\title{
Aortic sinus pouch technique for transposition of the great arteries with intramural coronary artery
}

\author{
Yoshihiro Ko, MD, Koji Nomura, MD, Hiroo Kinami, MD, and Ren Kawamura, MD, Saitama, Japan
}

\author{
From the Department of Cardiovascular Surgery, Saitama Children's Medical Center, Saitama, Japan. \\ Disclosures: Authors have nothing to disclose with regard to commercial support. \\ Received for publication Oct 12, 2017; accepted for publication Oct 22, 2017; available ahead of print Nov 29, \\ 2017. \\ Address for reprints: Yoshihiro Ko, MD, Department of Cardiovascular Surgery, Saitama Children's Medical Cen- \\ ter, 1-2 Shintoshin, Chuou-ku, Saitama 330-8777, Japan (E-mail: koyoshihiro@yahoo.co.jp). \\ J Thorac Cardiovasc Surg 2018; 155:e127-9 \\ $0022-5223 / \$ 36.00$ \\ Copyright (c) 2017 by The American Association for Thoracic Surgery \\ https://doi.org/10.1016/j.jtcvs.2017.10.073
}

Video clip is available online.

The arterial switch operation (ASO) for transposition of the great arteries (TGA) has been developed for many anatomic coronary artery variants, and numerous coronary transfer techniques have been reported. TGA with intramural coronary arteries, however, is still associated with increased risk. We achieved a good result by applying an aortic sinus pouch technique for dextro-TGA (d-TGA) with an intramural coronary artery.

\section{CLINICAL SUMMARY}

A boy was born at term and transferred to our institution for severe cyanosis. He had d-TGA with intact ventricular septum diagnosed. The coronary artery pattern was identified as Shaher $2 \mathrm{a}$, with the origin of the single left anterior descending artery (LAD) unclear on preoperative examination. Although the left ventricle was enlarged, ventricular pressure and function were acceptable. On day 13 after birth, weighing $2.9 \mathrm{~kg}$, he underwent ASO.

We were unable to confirm the origin of the LAD with only visual evaluation of the heart. Under cardiopulmonary bypass, the ascending aorta was transected $13 \mathrm{~mm}$ above the right ventricle. A small, slitlike orifice in the LAD was seen in the right-facing sinus near the right coronary artery orifice. The LAD followed an intramural route behind the posterior valvar commissure, and the arterial intima along the route was thick and hard. We therefore determined that a coronary direct implantation presented a high risk, and instead we applied an aortic sinus pouch technique. We detached the posterior valvar commissure and unroofed the small orifice in the LAD up to $1.5 \mathrm{~mm}$. Next, we cut the left- and right-facing sinuses away from the aortic Valsalva and sutured the edge of the aortic sinus

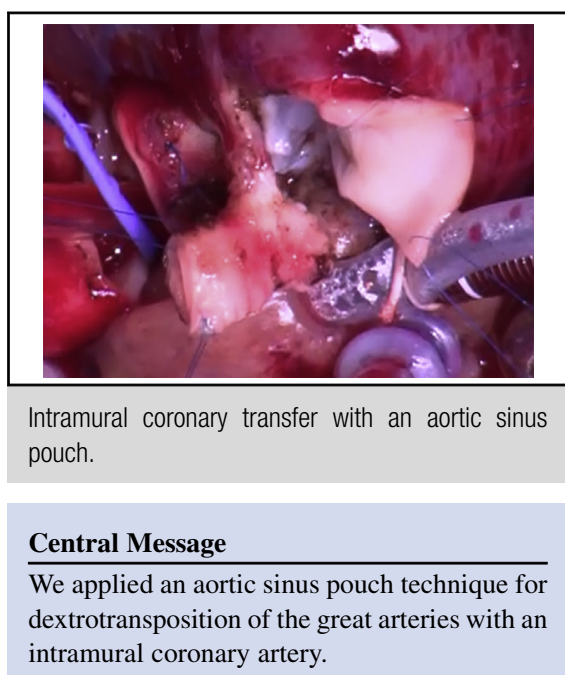

See Editorial Commentaries pages e131 and e133.

button to create a long tubular pouch on the proximal end of the coronary trunk. The pulmonary artery was then transected just below the bifurcation. A triangular tip of the main pulmonary trunk was resected, and the pouch was cut $5 \mathrm{~mm}$ vertically and anastomosed to the pulmonary trunk in a double-barreled manner (Figure 1 and Video 1). The ascending aorta was reconstructed with the LeCompte maneuver, and the pulmonary artery was reconstructed with fresh pericardium. The atrial septal defect was closed directly. The patient had an uneventful postoperative course, and postoperative computed tomography indicated a tubular pouch on the proximal end of the coronary trunk, which separated from the pulmonary arterial wall (Figure 2).

\section{DISCUSSION}

ASO is the criterion standard operation for d-TGA; however, the presence of an intramural coronary artery is still associated with increased risk. The incidence of intramural coronary arteries in TGA ranges from $3 \%$ to $5 \%,{ }^{1}$ and they need to be kept in mind in ASO because they may not be apparent until the aorta has been opened. Intramural coronary arteries often impede coronary button sampling during ASO. In most cases, the intramural coronary artery originates from the wrong sinus, resulting in single coronary ostia or kissing edges. In addition, the intramural route or 

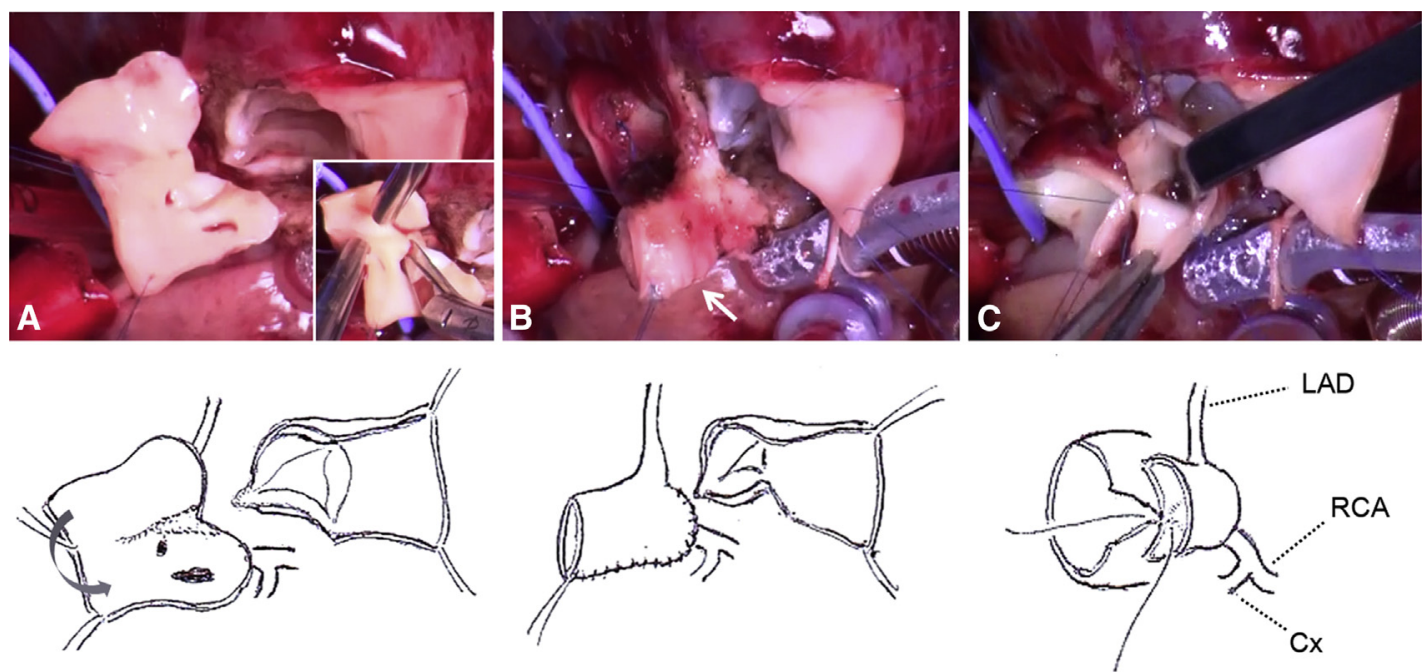

FIGURE 1. Operative photographs and diagrams. A, Two coronary orifices in the right-facing sinus; removal of the left- and right-facing aortic sinuses from the aortic trunk after detaching the posterior commissure and unroofing the intramural coronary ostium (inset). B, The aortic sinus pouch is the long tubular pouch on the proximal end of the main coronary trunk. C, Double-barreled anastomosis is completed between the aortic sinus pouch and the pulmonary trunk. $L A D$, Left anterior descending coronary artery; $R C A$, right coronary artery.

the ostium between the great arteries may be close to the posterior valvular commissure.

We previously reported on our aortic sinus pouch technique for ASO with a single coronary artery, ${ }^{2}$ and at the time we believed that we might also be able to apply the technique for ASO with intramural coronary. It may solve most of problems associated with other previously reported techniques, such as aortopulmonary fenestration with an

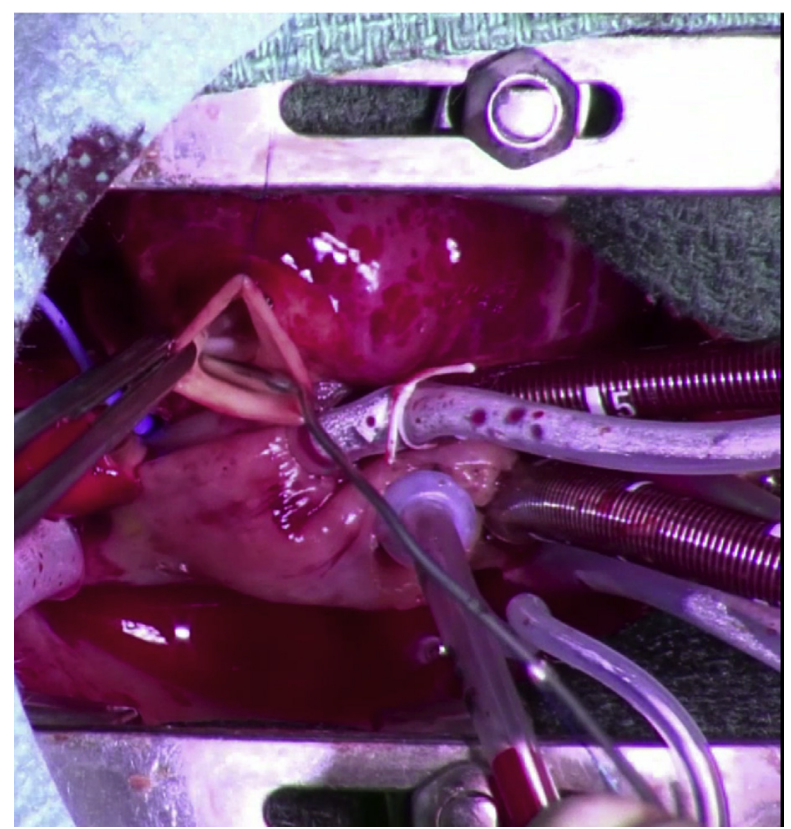

VIDEO 1. Intramural coronary artery transfer technique with an aortic sinus pouch. Video available at: http://www.jtcvsonline.org/article/S00225223(17)32402-9/fulltext. aortic wall flap or pericardial baffle $e^{3,4}$ or coronary direct implantation, ${ }^{5}$ because the coronary artery and the ostia are left in the original anatomic position, and the proximal coronary route is completely separate from the great arteries. Furthermore, the length of the proximal coronary pouch and the direction of its anastomosis to the pulmonary trunk can be regulated regardless of the site of the coronary ostia. For these reasons, this technique might be a useful option for ASO with any complex coronary anatomy taking off from a single aortic sinus. It should be noted, however, that one of the ischemic mechanisms of an intramural

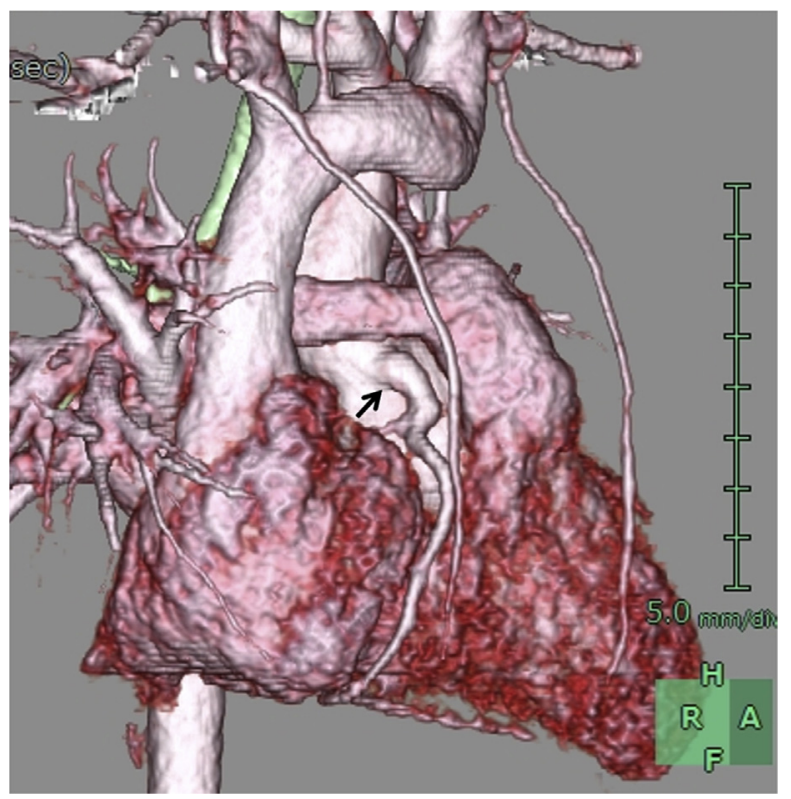

FIGURE 2. Postoperative computed tomography. The aortic sinus pouch (arrow) is separated from the pulmonary artery. 
coronary artery is abnormal vascular wall structure leading to stenosis of the intramural segment, ${ }^{1}$ and that if full unroofing of the coronary ostium can be performed, the distal portion of the intramural segment where the coronary artery leaves the aortic wall cannot be opened and remains potentially stenotic, regardless of technique.

In conclusion, the technique presented in this report is simpler and safer than previously reported procedures. To our knowledge, this is the first report of a coronary transfer technique to use an aortic sinus pouch for d-TGA with intramural coronary artery.

\section{References}

1. Metton O, Calvaruso D, Gaudin R, Mussa S, Raisky O, Bonnet D, et al. Intramura coronary arteries and outcome of neonatal arterial switch operation. Eur J Cardiothorac Surg. 2010;37:1246-53.

2. Ko Y, Nomura K, Nakao M. New coronary transfer technique for transposition of the great arteries with a single coronary artery. J Thorac Cardiovasc Surg. 2017; 153:1150-2.

3. Aubert J, Pannetier A, Couvelly JP, Unal D, Rouault F, Delarue A. Transposition of the great arteries. New technique for anatomical correction. Br Heart J. 1978;40: 204-8.

4. Moat NE, Pawade A, Lamb RK. Complex coronary arterial anatomy in transposition of the great arteries. Arterial switch procedure without coronary relocation. $J$ Thorac Cardiovasc Surg. 1992;103:872-6.

5. Asou T, Karl TR, Pawade A, Mee RB. Arterial switch: translocation of the intramural coronary artery. Ann Thorac Surg. 1994;57:461-5. 